Dear Author,

Please, note that changes made to the HTML content will be added to the article before publication, but are not reflected in this PDF.

Note also that this file should not be used for submitting corrections. 
Review

\title{
Calcium signaling in human pluripotent stem cells
}

\author{
Q1 Ágota Apáti ${ }^{\mathrm{a}, *}$, Tünde Berecz ${ }^{\mathrm{a}}$, Balázs Sarkadi ${ }^{\mathrm{a}, \mathrm{b}}$ \\ a Institute of Enzymology, Research Centre for Natural Sciences, Hungarian Academy of Sciences, Budapest, Hungary \\ ${ }^{\mathrm{b}}$ MTA-SE Molecular Biophysics Research Group, Semmelweis University, Institute of Biophysics and Radiation Biology, Budapest, Hungary
}

\section{A R T I C L E I N F O}

\section{Article history:}

Received 8 October 2015

Received in revised form 14 January 2016

Accepted 19 January 2016

Available online $\mathrm{xxx}$

\section{Keywords:}

Calcium signals

Pluripotent stem cells

Pluripotency maintenance

Naïve and primed state

Calcium sensitive dyes

Genetically engineered calcium indicators

\begin{abstract}
A B S T R A C T
Human pluripotent stem cells provide new tools for developmental and pharmacological studies as well as for regenerative medicine applications. Calcium homeostasis and ligand-dependent calcium signaling are key components of major cellular responses, including cell proliferation, differentiation or apoptosis. Interestingly, these phenomena have not been characterized in detail as yet in pluripotent human cell sates. Here we review the methods applicable for studying both short- and long-term calcium responses, focusing on the expression of fluorescent calcium indicator proteins and imaging methods as applied in pluripotent human stem cells. We discuss the potential regulatory pathways involving calcium responses in hPS cells and compare these to the implicated pathways in mouse PS cells. A recent development in the stem cell field is the recognition of so called "naïve" states, resembling the earliest potential forms of stem cells during development, as well as the "fuzzy" stem cells, which may be alternative forms of pluripotent cell types, therefore we also discuss the potential role of calcium homeostasis in these PS cell types.
\end{abstract}

(C) 2016 Published by Elsevier Ltd.

\section{Introduction}

Human pluripotent stem cell (hPSC) lines can be established from the inner cell mass of blastocyst (embryonic stem cells-hESC [1]) or by reprograming various somatic cells by using key transcription factors (induced pluripotent stem cells-hiPSC [2]). In addition, hPSCs can be generated with normal genetic background or with patient-specific mutations, depending on the starting cell

Abbreviations: hPSC, human pluripotent stem cell; mPSC, mouse pluripotent stem cell; hESC, human embryonic stem cell; iPSC, induced pluripotent stem cell; GECI, genetically engineered calcium indicator; EGF, epidermal growth factor; FGF, fibroblast growth factor; FGFR, fibroblast growth factor receptor; IGF, insulinlike growth factor; TGF $\beta$, ransforming growth factor $\beta$; Wnt, Wingless/Integrated; GPCR, G protein-coupled receptor; ERK, extracellular signal-regulated kinase; PI3K, phosphoinositide 3-kinase; MEK, MAPK/ERK kinase; MAPK, mitogen-activated protein kinase; PLC $-\gamma$, phospholipase C $-\gamma$; mTOR, mammalian target of rapamycin; FZ, frizzled; DVL, disheveled; PKC, protein kinase C; GSK3, glycogen synthase kinase 3; LPA, lysophosphatidic acid; SERCA, sarcoplasmic/endoplasmic calcium ATPase; CPA, cyclopiazonic acid; TG, thapsigargin; CAMKII, calcium/calmodulindependent protein kinase II; AP, alkaline phosphatase; GABA, $\gamma$-aminobutyric acid; LIF, leukemia inhibitory factor; JAK, Janus kinase; STAT, signal transducer and activator of transcription; BMP, bone morphogenetic protein; HERVH, human endogenous retrovirus $\mathrm{H}$.

Q2 * Corresponding author at: Institute of Enzymology, Research Centre for Natural Sciences, Hungarian Academy of Sciences, Magyar tudósok krt. 2, Budapest 1117, Hungary. Fax: +3613826295.

E-mail addresses: apati@biomembrane.hu, apati.agota@gmail.com (Á. Apáti). populations [3,4]. Irrespective of the source and procedure they are generated, all hPSCs can be cultured practically for unlimited time in vitro, and have the potential to differentiate into all cell types (such as cardiomyocytes, hepatocytes, neural cell types etc.) of human tissues and organs [5,6]. Therefore hPSCs represent unique models for developmental studies or drug screening, and hopefully also provide the basis of future cell-based medical therapies.

Calcium signaling studies have been performed in a great number by using hPSC-derived, differentiated cell types (especially neurons and cardiomyocytes) [7,8], while there are only limited data on calcium signaling in pluripotent stem cells. In the current paper we provide an overview of the methodologies currently applied for such studies, summarize the signal transduction pathways involving calcium movements in hPSCs, discuss the differences between mouse and human PSCs in these regards, and briefly survey the potential signaling differences in the newly emerging alternative PSC states.

\section{Methods for studying calcium signals in human PS cells}

Currently the most widely applied methods for studying calcium signaling pathways use fluorescent indicators combined with imaging methods, allowing these investigations with a minimum perturbance of the physiological cellular function. The applied imaging methods include a great variety of fluorescence microcopy, flow cytometry, confocal and two-photon microscopy techniques, 
54 while the basis of all these methods is the introduction of fluorescent calcium indicators into the living cells.

The application of small molecule, cell permeable synthetic calcium indicators brought a breakthrough in studying cellular calcium signaling (see Refs. $[9,10])$. These calcium sensitive dyes have been and are still widely used in in vitro calcium signaling studies, while their applications have numerous drawbacks. Loading of the cells requires special conditions, and in many cases the liberation of the calcium indicator dye from the cell permeable compound generates toxic metabolites. Intracellular dye accumulation may significantly alter signaling by providing a calcium buffer system. The reproducibility of the loading procedure has to be carefully controlled, and the distribution of the dye between cells and inside particular cell compartments may cause major problems. In addition, dye quenching and/or bleaching may occur during extended imaging periods (for details see Refs. [11,12]), and an in vivo application of these dyes is strongly limited.

Genetically engineered calcium indicator proteins (GECIs) address many of these limitations and serve as alternatives of calcium sensitive dyes in signaling studies. The development of GECIs resulted in the generation of a wide range of indicator proteins with different optical properties. In addition, by using tissue specific promoters and selective cellular targeting modifications, these proteins became most useful for calcium signaling analysis within selected tissues, specific cell types or intracellular compartments (see Refs. [13-17]). In most cases, under proper expression conditions, GECIs have minimum effects on cellular activity, can be used in vivo as well, and therefore are also suitable for long-term studies (see Ref. [18]), although potential calcium buffering and cellular signal perturbations may still occur ([19]) (as reviewed in Refs. [20,21]).

As a summary, calcium sensitive small molecular dyes have several advantages over GECIs, including faster responses, near-linear responses, and higher sensitivities [22,23]. In contrast, GECIs can be better targeted into specific cell types or specific cell compartments, allow long term measurements, and have better signal to noise ratios at least in in vivo experiments [22]. A recent development of GECIs has resulted in a new class of indicator proteins, including GCaMP6, with higher sensitivity and detection reliability even for individual action potentials in neurons [24]. Thus, depending on the actual scientific question, investigators should choose probes most suitable for a given application, considering response kinetics, probe sensitivity, signal to noise ratio and other aspects of the experimental goals. While cells and tissues differentiated from PSCs have been widely used for calcium signaling studies, we found only a limited number of data about such signaling in pluripotent stem cells, and even these data are mainly from mouse cell studies. Calcium signals in hESC-derived cardiomyocytes and neural cell types have been measured in most cases by calcium sensitive dyes: for cardiomyocytes Fura-2/AM [25-28], Fluo-4/AM [29,30] or dsRed+ [27], while for neural cell types Fluo3/AM [8,31] Fura-2/AM [8,32] or Oregon green 488 Bapta-1 AM [33] have been applied. In pluripotent cells the application of Fluo3/AM (mESC-[34], hESC-[8]) or Fluo-4/AM (mESC-[35-37], and hESC [12]) has been reported. The application of GECIs, while already widely reported in various functional studies, has only been described in mPSCs [38] and in hPSCs, as well as in their differentiated offsprings (see Apati et al. [39]).

In our recent studies [39], we have compared in detail the application of the small molecule calcium sensitive fluorescent dye, Fluo-4, and a GECI, GCaMP2, for studying calcium signaling in human PSCs. We essentially found that the differentiation properties were fully preserved in the cloned GCaMP2-expressing hESC lines, and calcium imaging could be performed without the need of any, potentially toxic dye-loading of the cells. Calcium signaling measurements performed in our laboratory in such GCaMP2 expressing hPS cells, as compared to signaling in Fluo4 loaded hPSCs, are shown in Fig. 1. As documented in Fig. 2, we have also observed a more uniform distribution of GCaMP2 in the transgenic, cloned human PSCs than in Fluo-4 dye-loaded preparations, while a major difference was observed regarding the subcellular distribution of the calcium signals evoked by extracellular ligands. As demonstrated, in Fluo-4 loaded cells the calcium signals were the greatest in the nuclear areas, while in the GCaMP2 expressing cells the cytoplasmic sensor protein gave the major signal (Fig. 2). As discussed in the literature, it is unclear if a specific dye distribution or the difference in the fluorescence properties is responsible for the high nuclear signal observed when using small molecule calcium sensors [40,41]. Still, it is a major advantage of the GCaMP2 protein that such a non-specific effect does not occur.

\section{Signaling pathways involved in pluripotency state maintenance and differentiation induction; possible role of calcium-dependent pathways}

There are numerous factors involved in the maintenance of the human stem cell pluripotent state. The applied culturing conditions and compounds used for maintaining pluripotency significantly affect the basal activity of various signaling and effector pathways, and the evident crosstalk between these signaling pathways renders even more difficult to elucidate the role of any individual route. The potential heterogeneity of pluripotent stem cell subpopulations (see Section 4) further complicates this picture. Still, by now there is a generally accepted concept about the key signaling pathways involved in the maintenance of human stem cell pluripotency.

Detailed analysis of different culturing conditions [42-44] have documented that the key ligands and receptors which are involved in the maintenance of the pluripotent state of human ESCs are (A) FGF2 and IGF trough tyrosine kinase receptors, (B) TGFb ligands (including TGFb, Nodal and Activin A) acting through the TGFb receptor family, and (C) Wnt ligands trough GPC receptors and other GPCRs with tyrosine kinase activity. Comparative analysis of the human and mouse embryonic stem cell transcriptomes confirmed that the components of these pathways are expressed at high levels in the hPSCs (see below and $[45,46]$ ). In all these pathways, changes in cytoplasmic calcium concentrations may have significant modulating effects.

A.) Signals evoked by FGF2 seem to be indispensable for maintaining hSC pluripotent state. FGF2, in combination with three small molecule inhibitors, supports the long-term maintenance of pluripotent hESC cultures [47], while blocking the FGF receptor signaling leads to rapid differentiation of these cells $[48,49]$ In undifferentiated hPS cells FGF2 acts directly on FGFR by stimulating signaling pathways downstream of the receptor [50,51], as well as indirectly by modulating signals in feeder cells or in the neighboring hPSC-derived niche cells [52-55]. Direct action of FGF2 on FGF receptors initiates several coupled signaling events, as summarized in Fig. 3.

The effect of FGF2 is concentration dependent on ERK activation and on the consequent cellular responses (see review by Dalton [56]). Low levels of FGF2 cause a mild elevation of ERK activity and does not affect the PI3K/AKT pathway, while higher doses of FGF2 activate both pathways, and the activation of PI3K/AKT results in a cross-inhibition on MEK and ERK, reducing ERK activity [51] (Fig. 3). The balanced, moderate level of activated ERK sustains the pluripotent state [51,57], while a strong reduction of PI3K activity causes elevated ERK activity followed by differentiation of PSCs (as shown in mouse studies $[58,59])$. 

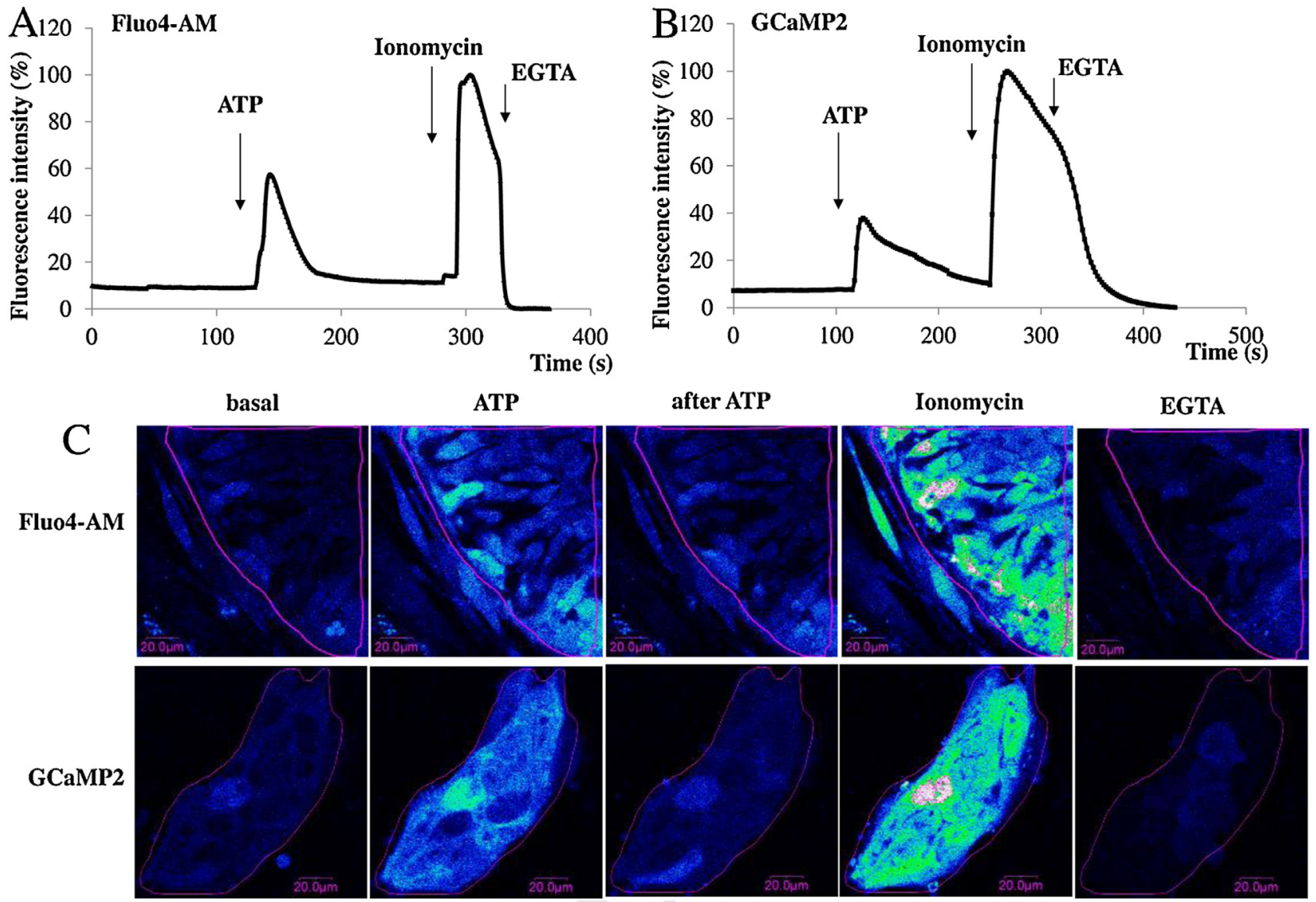

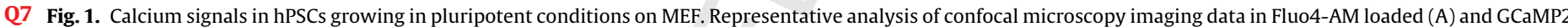

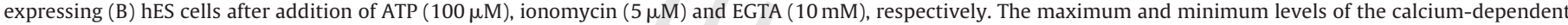

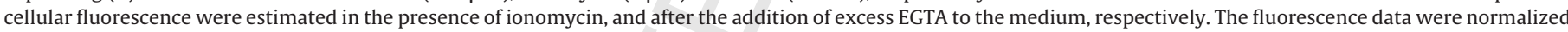

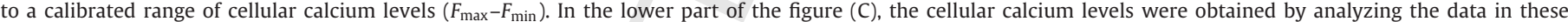
measurements (Apati et al., unpublished experiments).

Since FGF2 also induces IGF-II production in feeder or hESCderived fibroblast-like cells, the generated IGF-II may modulate ERK activity by activating the PI3K/AKT signal through the IGF receptor (Fig. 3) [52,56]. While the MAPK and PI3K/AKT pathways induced by FGF2 have been extensively studied in hPSCs, there are only limited data for other potential signaling events. Examination of tyrosine phosphorylation profiles in hESCs, stimulated by FGF-2, demonstrated an increase in tyrosine phosphorylation of all four FGFRs, and also some of their canonical downstream effectors (eg. PLC- $\gamma$, MAPK, PI3-K). In addition, a large number of pY-peptides, not directly involved in the canonical FGF pathway (e.g., Src kinase substrates, additional receptor tyrosine kinases and others), were observed following FGF-2 stimulation [60]. This quantitative phosphoproteomic analysis suggests that intracellular calcium modulation may become important through the activation of the PLC- $\gamma$ pathway (Fig. 3), and an increase in cytoplasmic calcium may further modulate the crosstalk between growth-factor dependent signaling events. We found no experimental data as yet for calcium signals evoked by FGF2 or IGF II in hPSCs.

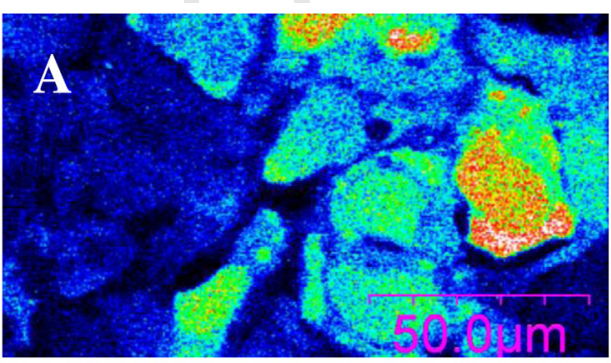

Fluo-AM

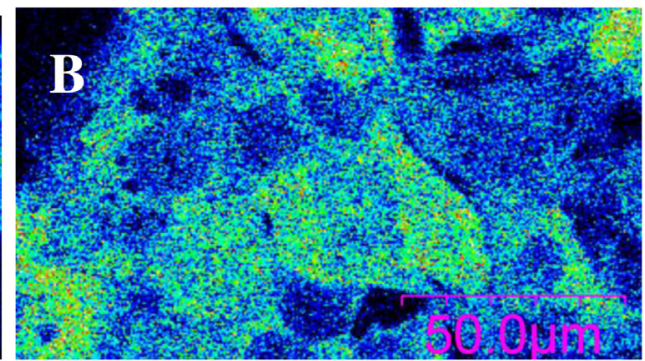

GCaMP2

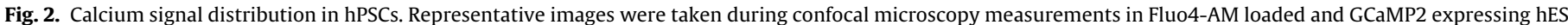

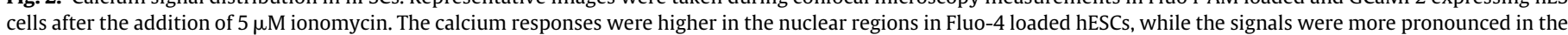
cytoplasmic area in GCaMP2 expressing hESCs. Moreover, Fluo-4 dye distribution among the cells was non-uniform (Apati et al., unpublished experiments).

Please cite this article in press as: Á. Apáti, et al., Calcium signaling in human pluripotent stem cells, Cell Calcium (2016), http://dx.doi.org/10.1016/j.ceca.2016.01.005 


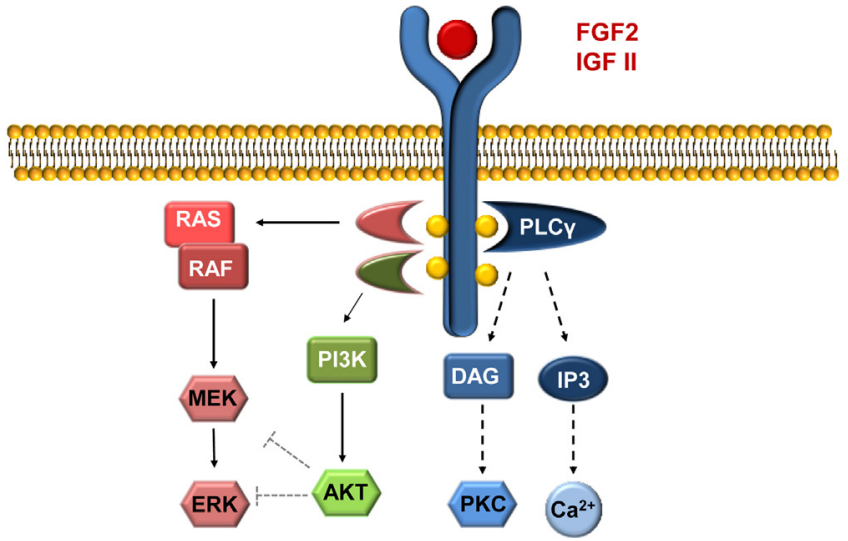

Fig. 3. Key components of the EGF and IGF receptor dependent pathways in PSCs. The Ras-MAP kinase pathway: binding of ligands to the receptor monomers causes dimerization and autophosphorylation of tyrosine residues. Ras activation occurs by coupling to the receptor through adaptor proteins and exchange of GDP for GTP. Ras binds to Raf and activates GTP hydrolysis to GDP. Raf activates MEK and MEK activates ERK at the expense of ATP hydrolyzation. IP3/DAG pathway: Phospholipase $\mathrm{C}-\gamma(\mathrm{PLC}-\gamma)$ is activated by the active tyrosine kinase receptor. Hydrolyzation of phosphatidylinositol 4,5-bisphosphate (PIP2) by PLC- $\gamma$ produces two distinct second messengers; inositol 1,4,5-trisphosphate (IP3) and diacylglycerol (DAG). IP3 binds to IP3 receptor, localized at the endoplasmic reticulum (ER) and induces $\mathrm{Ca}^{2+}$ release from the ER. Meanwhile DAG, remaining in the plasma membrane, activates protein kinase $C$ (PKC). PI3-kinase pathway: Activated phosphoinositide 3-kinase (PI3-K) activates AKT by phosphorylation. AKT inhibits the activation of MEK and ERK by suppressing Raf kinase activity.

(A.) Activin A $[43,61]$ and the other members of the TGF $\beta$ ligand family (TGF $\beta$, Nodal) [62] have been shown to support the maintenance of pluripotent state under feeder-cell free conditions. The TGF $\beta$ family members, through the activation of TGFb receptor and subsequently Smad 2, 3, initiate the transcriptional activation of NANOG, and generate a well characterized gene product for pluripotency. While there are numerous data for a cross-talk between the PI3K/AKT-mTOR, MAPK and SMAD2,3 signaling pathways (reviewed in Ref. [56]), the involvement of calcium signaling in this crosstalk has not yet been documented.

(B.) The extracellular signaling molecule Wnt interacts with the transmembrane receptor frizzled (FZ), a G protein coupled receptor (GPRC), and the signal transducer disheveled (DVL) induces three independent signaling pathways (Fig. 4). The canonical pathway leads to $\beta$-catenin translocation into the nucleus and activation of target genes. The planar cell polarity pathway through the activation of Rho-GTPases induces mostly changes in the cytoskeleton. The WNT/calcium pathway activates protein kinase C (PKC) and elevates the cytoplasmic calcium level, which in turn, activates various calcium/calmodulin-dependent proteins [63]. A recent study has shown that several Wnt ligands increase cytosolic and intranuclear $\mathrm{Ca} 2+$, and facilitate $\beta$-catenin entry into the nucleus [64].

There are contradicting data regarding the role of Wnt signaling in maintaining pluripotency in hPSCs. Several published papers indicate the role of Wnt in supporting pluripotency [65,66], while other data emphasize Wnt signaling in hPSC differentiation [67]. Recently it has been suggested that different GSK3 pools may play different roles in the control of hPSCs fate determination [56].

In addition to the receptor FZ for Wnt signaling, there are other GPCRs which may play a role in pluripotent cell fate. As shown in Fig. 5, a combined action of selected GPCRs may occur through calcium independent and calcium-dependent pathways as well.

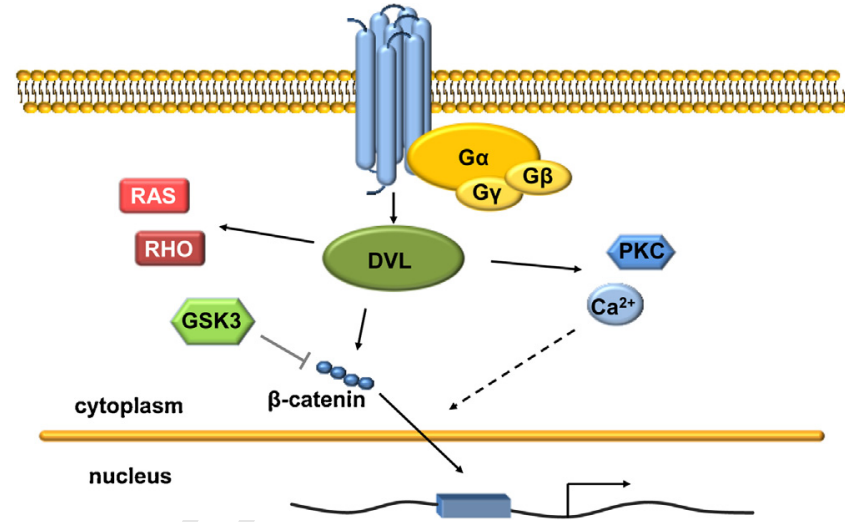

Fig. 4. Wnt signaling pathways potentially involved in PSC maintenance and differentiation. Canonical Wnt pathway: Wnt ligand binds to Frizzled (Fz) receptor The activated receptor forms a complex with the co-receptor LRP5/6. Disheveled (DVL) is phosphorylated thus activated. In absence of Wnt, APC/Axin/GSK-3 complex causes the proteosomal degradation of $\beta$-catenin. Activated DVL inhibits this complex, resulting in the accumulation of $\beta$-catenin. $\beta$-catenin is translocated to the nucleus and activates gene expression. (2) Non-canonical planar cell polarity pathway: activated DVL forms a complex with an activator resulting in the activation of Rho small G-protein. Ras is also activated by DVL and with the activated Rho induces cytoskeleton regulation. (3) Non-canonical Wnt/calcium pathway: In this pathway DVL is activated by Fz receptor directly and also through a G-protein. DVL activates protein kinase $\mathrm{C}(\mathrm{PKC})$ and $\mathrm{Ca}^{2+}$ release from ER through PLC activation. $\mathrm{Ca}^{2+}$ may contribute to the translocation of $\beta$-catenin to the nucleus.

Ermakov et al. [68] suggested that an elevation of intracellular calcium may have a significant role in the fate of hPSCs. They found that an elevation of intracellular calcium, induced by the GPCR receptor agonist LPA, or by SERCA pump inhibitors (CPA, TG and chlorpromazine or clotrimazole), can substitute FGF2 under 
feeder-free hPSC culturing conditions. According to this work, the inhibition of the CAMKII kinase by the specific inhibitor KN-62 resulted in a decrease in alkaline phosphatase positive (AP+) colony number, suggesting a role of CAMKII kinase in maintaining the pluripotent state [68]. Still, the actual calcium signals evoked by LPA or SERCA pump inhibitors in hPSCs have not been demonstrated.

In our recent studies cellular calcium signals induced by various ligands of GPCRs (ATP, LPA, trypsin or angiotensin II) in undifferentiated hES cells have been demonstrated, both in GCaMP2 expressing and in Fluo-4 loaded cells. In these experiments we found no calcium signal evoked by other potential ligands, e.g., thrombin, histamine or GABA [39].

While this current paper focuses on calcium signals in pluripotent state, there is an emerging new field related to potential therapeutic applications of hPSC derived neural cell types, which exhibit essential calcium signals. As Viero et al. [69] pointed out, transplantation of hESC derived neural cell types is difficult and ineffective; therefore the best option for cell therapy remains the use of neural precursor cells (NPCs). Generation of suitable human NPCs for neural differentiation and further transplantation is challenging because of the lack of standardized protocols and functional studies. Forostyak et al. [32] and Viero et al. [69] demonstrated that calcium signals in hPSC derived NPCs may reflect their regeneration capacity after transplantation. These NPCs exhibited spontaneous calcium oscillations and calcium signals evoked by high $\mathrm{K}+$, ATP, glutamate, g-aminobutyric acid (GABA) and caffeine, indicating that the electrophysiological and calcium handling properties of NPCs are similar to those of matured neurons. These authors also showed that calcium signals depend on culturing conditions, e.g., the passage number of culture, and the NPCs with the highest activity of calcium signaling yielded the best results in terms of functional improvement after transplantation. Similar Ca2+ signaling patterns were found when an immortalized neural stem cell line (SPC-01 derived from human fetal spinal cord) was studied [70], showing functional L-and $\mathrm{P} / \mathrm{Q}$-typeCa2+ channels and the occurrence of spontaneous $\mathrm{Ca} 2+$ oscillations. Recently our group has also established NPCs from iPS cells stably expressing GCaMP calcium indicator proteins and found this system most relevant for studying calcium signaling (unpublished studies).

A considerable problem in studying calcium signals in pluripotent stem cells is a potentially major difference in the relevant pathways between the most studied mouse PSCs (MPSC) and their human counterparts. While in MPSCs the presence of LIF, acting through the JAK-STAT pathway, and BMP, acting through a SMAD-dependent pathway, are supporting the pluripotency state, in hPSCs LIF is not required and BMP-SMAD induce cell differentiation. FGF2 and related calcium signaling in hPSCs maintains pluripotency, while FGF2 and PI3K activation, mostly acting through the elevation of cellular calcium, induce differentiation in mPSCs (for details see Refs. [45,46,71]). Clearly, further studies are required in both PS cell types to clarify the role of calcium signaling in the maintenance of pluripotency and the induction of directed stem cell differentiation.

\section{Alternative pluripotency cell states - from naïve to fuzzy - potential role and characteristics of signaling pathways}

Based on mammalian, mostly mouse embryology reprogramming studies, "naïve-state" pluripotent stem cells have been claimed different from the "non-naïve" or "primed" PSCs - as first suggested by Nichols and Smith in 2009 [72] - by several criteria. According to this concept, in the pre-implantation embryos the pluripotent stem cells are 'naïve', but they become 'primed' during post-implantation development, and most of the currently available ES or iPS cells correspond to this latter state. While both cell types express pluripotency markers, form teratomas in-vivo and can differentiate into mature cells of all the three germ layers, naïve hPSCs express substantially reduced epigenetic markers, survive single-cell cloning and may form cross-species chimeras. Naïve PSCs are also characterized by e.g., driving Oct4 transcription by a distal enhancer, having a non-activated X chromosome state, and major reduction in DNA methylation (see Refs. [72-74]).

As we discussed above, mouse and human PSCs may be quite different in the signaling pathways maintaining pluripotency. Still, there are numerous, reportedly successful efforts to convert primed human PSCs to a naïve state [75-79], as well as to elucidate the signaling pathways that regulate these different states of pluripotency. In a recent work, Wang et al. [77] documented the presence of a "naïve-like" subpopulation in human ES and iPS cells, showing an elevated transcription of HERVH, a primate-specific endogenous retrovirus, as a hallmark of this state.

The naïve state of pluripotency has been suggested to provide a much broader and efficient developmental potential as compared to the primed cell types. However, there is very little information as yet regarding the signal transduction differences between naïve and primed human stem cells. One report [78] documents a reverse "toggling" of primed hESC lines by the addition of histone deacetylase inhibitors, and subsequently MEK/ERK and GSK3 inhibitors, as well as FGF2. In addition, this report also demonstrates a direct derivation of naïve PS cells from a human embryo by using MEK/ERK and GSK3 inhibitors together with FGF2. Similarly, a combined inhibition and alteration of these relevant signaling pathways was used by Gafni et al. [75], to generate human naïve-like ES cells.

These data, in combination with the above described potential role of the MEK/ERK pathway and growth factors in altering the calcium homeostasis in the pluripotent cells, may raise the role of calcium in these "toggling" experiments. However, we found no report directly concerned with studying the relevant calcium signaling pathways either in the naïve of the "fuzzy" pluripotent stem cells. The Project Grandiose: high definition characterization of the process of reprogramming cells to pluripotency (www.stemformatics.org), provides a detailed analysis of pluripotent stem cell variants regarding their proteome, RNA transcriptome, epigenome-methylome (epigenetic marks), microRNA + long non-coding RNA, extended with the integration of proteome, transcriptome and epigenome data (www. stemformatics.org), thus hoping to provide a molecular encyclopedia of reprogramming. Still, regulation of the signaling pathways is not included in this analysis. We hope that future research will concentrate on these absolutely essential cellular phenomena as well.

\section{Acknowledgements}

This work has been supported by the Hungarian Scientific Q5 Research Fund [K115375]; Hungarian Brain Research Program [KTIA VKSZ_12, NAP-A-1.10] and by the National Research, Development and Innovation Office [KTIA_AIK_12-1-2012-0025, KMR_12-1-2012-0112].

\section{References}

[1] J.A. Thomson, J. Itskovitz-Eldor, S.S. Shapiro, et al., Embryonic stem cell lines derived from human blastocysts, Science 282 (1998) 1145-1147.

[2] K. Takahashi, S. Yamanaka, Induction of pluripotent stem cells from mouse embryonic and adult fibroblast cultures by defined factors, Cell 126 (2006) 663-676.

[3] M. Bellin, M.C. Marchetto, F.H. Gage, C.L. Mummery, Induced pluripotent stem cells: the new patient? Nat. Rev. Mol. Cell Biol. 13 (2012) 713-726.

[4] M. Ohnuki, K. Takahashi, Present and future challenges of induced pluripotent stem cells, Philos. Trans. R. Soc. Lond. B Biol. Sci. 370 (2015). 
[5] K.K. Hirschi, S. Li, K. Roy, Induced pluripotent stem cells for regenerative medicine, Annu. Rev. Biomed. Eng. 16 (2014) 277-294.

[6] J. Itskovitz-Eldor, M. Schuldiner, D. Karsenti, et al., Differentiation of human embryonic stem cells into embryoid bodies compromising the three embryonic germ layers, Mol. Med. 6 (2000) 88-95.

[7] S. Li, G. Chen, R.A. Li, Calcium signalling of human pluripotent stem cell-derived cardiomyocytes, J. Physiol. 591 (2013) 5279-5290.

[8] S. Malmersjo, I. Liste, O. Dyachok, A. Tengholm, E. Arenas, P. Uhlen, Ca2+ and cAMP signaling in human embryonic stem cell-derived dopamine neurons, Stem Cells Dev. 19 (2010) 1355-1364.

[9] C. Stosiek, O. Garaschuk, K. Holthoff, A. Konnerth, In vivo two-photon calcium imaging of neuronal networks, Proc. Natl. Acad. Sci. U. S. A. 100 (2003) 7319-7324.

[10] R. Yuste, A. Peinado, L.C. Katz, Neuronal domains in developing neocortex, Science 257 (1992) 665-669.

[11] R.M. Paredes, J.C. Etzler, L.T. Watts, W. Zheng, J.D. Lechleiter, Chemical calcium indicators, Methods 46 (2008) 143-151.

[12] A. Apati, K. Paszty, Z. Erdei, K. Szebenyi, L. Homolya, B. Sarkadi, Calcium signaling in pluripotent stem cells, Mol. Cell. Endocrinol. 353 (2012) 57-67.

[13] T. Hendel, M. Mank, B. Schnell, O. Griesbeck, A. Borst, D.F. Reiff, Fluorescence changes of genetic calcium indicators and OGB-1 correlated with neural activity and calcium in vivo and in vitro, J. Neurosci. 28 (2008) 7399-7411.

[14] S.A. Hires, L. Tian, L.L. Looger, Reporting neural activity with genetically encoded calcium indicators, Brain Cell Biol. 36 (2008) 69-86.

[15] J.E. McCombs, A.E. Palmer, Measuring calcium dynamics in living cells with genetically encodable calcium indicators, Methods 46 (2008) 152-159.

[16] Y.N. Tallini, J.F. Brekke, B. Shui, et al., Propagated endothelial Ca2+ waves and arteriolar dilation in vivo: measurements in Cx40BAC GCaMP2 transgenic mice, Circ. Res. 101 (2007) 1300-1309.

[17] L. Tian, S.A. Hires, T. Mao, et al., Imaging neural activity in worms, flies and mice with improved GCaMP calcium indicators, Nat. Methods 6 (2009) 875-881.

[18] K. Szebenyi, A. Furedi, O. Kolacsek, et al., Visualization of calcium dynamics in kidney proximal tubules, J. Am. Soc. Nephrol. (2015).

[19] K. Szebenyi, A. Furedi, O. Kolacsek, et al., Generation of a homozygous transgenic rat strain stably expressing a calcium sensor protein for direct examination of calcium signaling, Sci. Rep. 5 (2015) 12645

[20] M.I. Kotlikoff, Genetically encoded Ca2+ indicators: using genetics and molecular design to understand complex physiology, J. Physiol. 578 (2007) 55-67.

[21] M. Whitaker, Genetically encoded probes for measurement of intracellular calcium, Methods Cell Biol. 99 (2015) 153-182.

[22] A. Badura, X.R. Sun, A. Giovannucci, L.A. Lynch, S.S. Wang, Fast calcium senso proteins for monitoring neural activity, Neurophotonics 1 (2014) 025008.

[23] R.Y. Tsien, Fluorescent indicators of ion concentrations, Methods Cell Biol. 30 (1989) 127-156.

[24] T.W. Chen, T.J. Wardill, Y. Sun, et al., Ultrasensitive fluorescent proteins for imaging neuronal activity, Nature 499 (2013) 295-300.

[25] K. Dolnikov, M. Shilkrut, N. Zeevi-Levin, et al., Functional properties of human embryonic stem cell-derived cardiomyocytes: intracellular Ca2+ handling and the role of sarcoplasmic reticulum in the contraction, Stem Cells 24 (2006) 236-245.

[26] J. Liu, D.K. Lieu, C.W. Siu, J.D. Fu, H.F. Tse, R.A. Li, Facilitated maturation of $\mathrm{Ca} 2+$ handling properties of human embryonic stem cell-derived cardiomyocytes by calsequestrin expression, Am. J. Physiol. Cell Physiol. 297 (2009) C152-C159.

[27] J.D. Fu, P. Jiang, S. Rushing, J. Liu, N. Chiamvimonvat, R.A. Li, Na+/Ca2+ exchanger is a determinant of excitation-contraction coupling in human embryonic stem cell-derived ventricular cardiomyocytes, Stem Cells Dev. 19 (2010) 773-782.

[28] J. Liu, J.D. Fu, C.W. Siu, R.A. Li, Functional sarcoplasmic reticulum for calcium handling of human embryonic stem cell-derived cardiomyocytes: insights for driven maturation, Stem Cells 25 (2007) 3038-3044.

[29] I. Itzhaki, S. Rapoport, I. Huber, et al., Calcium handling in human induced pluripotent stem cell derived cardiomyocytes, PLoS One 6 (2011) e18037.

[30] J. Satin, I. Itzhaki, S. Rapoport, et al., Calcium handling in human embryonic stem cell-derived cardiomyocytes, Stem Cells 26 (2008) 1961-1972.

[31] E. Smedler, S. Malmersjo, P. Uhlen, Network analysis of time-lapse microscopy recordings, Front. Neural Circuits 8 (2014) 111.

[32] O. Forostyak, N. Romanyuk, A. Verkhratsky, E. Sykova, G. Dayanithi, Plasticity of calcium signaling cascades in human embryonic stem cell-derived neural precursors, Stem Cells Dev. 22 (2013) 1506-1521.

[33] G.S. Belinsky, C.L. Sirois, M.T. Rich, et al., Dopamine receptors in human embryonic stem cell neurodifferentiation, Stem Cells Dev. 22 (2013) $1522-1540$.

[34] E. Yanagida, S. Shoji, Y. Hirayama, et al., Functional expression of Ca2+ signaling pathways in mouse embryonic stem cells, Cell Calcium 36 (2004) 135-146.

[35] N. Kapur, G.A. Mignery, K. Banach, Cell cycle-dependent calcium oscillations in mouse embryonic stem cells, Am. J. Physiol. Cell Physiol. 292 (2007) C1510-C1518.

[36] S. Mamo, J. Kobolak, I. Borbiro, T. Biro, I. Bock, A. Dinnyes, Gene targeting and calcium handling efficiencies in mouse embryonic stem cell lines, World J. Stem Cells 2 (2010) 127-140.

[37] M. Schwirtlich, Z. Emri, K. Antal, Z. Mate, Z. Katarova, G. Szabo, GABA(A) and $\mathrm{GABA}(\mathrm{B})$ receptors of distinct properties affect oppositely the proliferation of mouse embryonic stem cells through synergistic elevation of intracellular Ca(2+), FASEB J. 24 (2010) 1218-1228.

[38] J.S. Meyer, G. Tullis, C. Pierret, K.M. Spears, J.A. Morrison, M.D. Kirk, Detection of calcium transients in embryonic stem cells and their differentiated progeny, Cell. Mol. Neurobiol. 29 (2009) 1191-1203.

[39] A. Apati, K. Paszty, L. Hegedus, et al., Characterization of calcium signals in human embryonic stem cells and in their differentiated offspring by a stably integrated calcium indicator protein, Cell. Signal. 25 (2013) 752-759.

[40] C. Perez-Terzic, L. Stehno-Bittel, D.E. Clapham, Nucleoplasmic and cytoplasmic differences in the fluorescence properties of the calcium indicator Fluo-3, Cell Calcium 21 (1997) 275-282.

[41] D. Thomas, S.C. Tovey, T.J. Collins, M.D. Bootman, M.J. Berridge, P. Lipp, A comparison of fluorescent $\mathrm{Ca} 2+$ indicator properties and their use in measuring elementary and global Ca2+ signals, Cell Calcium 28 (2000) 213-223.

[42] C. Xu, M.S. Inokuma, J. Denham, et al., Feeder-free growth of undifferentiated human embryonic stem cells, Nat. Biotechnol. 19 (2001) 971-974.

[43] G.M. Beattie, A.D. Lopez, N. Bucay, et al., Activin A maintains pluripotency of human embryonic stem cells in the absence of feeder layers, Stem Cells 23 (2005) 489-495.

[44] R.H. Xu, T.L. Sampsell-Barron, F. Gu, et al., NANOG is a direct target of TGFbeta/activin-mediated SMAD signaling in human ESCs, Cell Stem Cell 3 (2008) 196-206.

[45] A. Schnerch, C. Cerdan, M. Bhatia, Distinguishing between mouse and human pluripotent stem cell regulation: the best laid plans of mice and men, Stem Cells 28 (2010) 419-430

[46] C.L. Wei, T. Miura, P. Robson, et al., Transcriptome profiling of human and murine ESCs identifies divergent paths required to maintain the stem cell state, Stem Cells 23 (2005) 166-185.

[47] H. Tsutsui, B. Valamehr, A. Hindoyan, et al., An optimized small molecule inhibitor cocktail supports long-term maintenance of human embryonic stem cells, Nat. Commun. 2(2011) 167.

[48] P. Dvorak, D. Dvorakova, S. Koskova, et al., Expression and potential role of fibroblast growth factor 2 and its receptors in human embryonic stem cells, Stem Cells 23 (2005) 1200-1211.

[49] V.M. Ding, L. Ling, S. Natarajan, M.G. Yap, S.M. Cool, A.B. Choo, FGF-2 modulates Wnt signaling in undifferentiated hESC and iPS cells through activated PI3-K/GSK3beta signaling, J. Cell. Physiol. 225 (2010) 417-428.

[50] M.E. Levenstein, T.E. Ludwig, R.H. Xu, et al., Basic fibroblast growth factor support of human embryonic stem cell self-renewal, Stem Cells 24 (2006) 568-574.

[51] A.M. Singh, D. Reynolds, T. Cliff, et al., Signaling network crosstalk in human pluripotent cells: a Smad2/3-regulated switch that controls the balance between self-renewal and differentiation, Cell Stem Cell 10 (2012) 312-326.

[52] S.C. Bendall, M.H. Stewart, P. Menendez, et al., IGF and FGF cooperatively establish the regulatory stem cell niche of pluripotent human cells in vitro, Nature 448 (2007) 1015-1021.

[53] L. Eiselleova, K. Matulka, V. Kriz, et al., A complex role for FGF-2 in self-renewal, survival, and adhesion of human embryonic stem cells, Stem Cells 27 (2009) 1847-1857.

[54] L. Eiselleova, I. Peterkova, J. Neradil, I. Slaninova, A. Hampl, P. Dvorak, Comparative study of mouse and human feeder cells for human embryonic stem cells, Int. J. Dev. Biol. 52 (2008) 353-363.

[55] B. Greber, H. Lehrach, J. Adjaye, Fibroblast growth factor 2 modulates transforming growth factor beta signaling in mouse embryonic fibroblasts and human ESCs (hESCs) to support hESC self-renewal, Stem Cells 25 (2007) 455-464.

[56] S. Dalton, Signaling networks in human pluripotent stem cells, Curr. Opin. Cell Biol. 25 (2013) 241-246.

[57] A.S. Bernardo, T. Faial, L. Gardner, et al., BRACHYURY and CDX2 mediate BMP-induced differentiation of human and mouse pluripotent stem cells into embryonic and extraembryonic lineages, Cell Stem Cell 9 (2011) 144-155.

[58] F. Lanner, J. Rossant, The role of FGF/Erk signaling in pluripotent cells, Development 137 (2010) 3351-3360

[59] J. Nichols, J. Silva, M. Roode, A. Smith, Suppression of Erk signalling promotes ground state pluripotency in the mouse embryo, Development 136 (2009) 3215-3222.

[60] V.M. Ding, P.J. Boersema, L.Y. Foong, et al., Tyrosine phosphorylation profiling in FGF-2 stimulated human embryonic stem cells, PLoS One 6 (2011) e17538.

[61] L. Xiao, X. Yuan, S.J. Sharkis, Activin A maintains self-renewal and regulates fibroblast growth factor, Wnt, and bone morphogenic protein pathways in human embryonic stem cells, Stem Cells 24 (2006) 1476-1486.

[62] T.E. Ludwig, V. Bergendahl, M.E. Levenstein, J. Yu, M.D. Probasco, J.A. Thomson, Feeder-independent culture of human embryonic stem cells, Nat.Methods 3 (2006) 637-646.

[63] Y. Komiya, R. Habas, Wnt signal transduction pathways, Organogenesis 4 (2008) 68-75.

[64] C. Thrasivoulou, M. Millar, A. Ahmed, Activation of intracellular calcium by multiple Wnt ligands and translocation of beta-catenin into the nucleus: a convergent model of Wnt/Ca2+ and Wnt/beta-catenin pathways, J. Biol. Chem. 288 (2013) 35651-35659.

[65] K.C. Davidson, A.M. Adams, J.M. Goodson, et al., Wnt/beta-catenin signaling promotes differentiation not self-renewal, of human embryonic stem cells and is repressed by Oct4, Proc. Natl. Acad. Sci. U. S. A. 109 (2012) 4485-4490.

[66] N. Sato, L. Meijer, L. Skaltsounis, P. Greengard, A.H. Brivanlou, Maintenance of pluripotency in human and mouse embryonic stem cells through activation of

\section{5}

455
455

$$
\begin{array}{r}
457 \\
458
\end{array}
$$

$$
\begin{array}{r}
459 \\
460
\end{array}
$$

\section{462
463}

\section{4}

\section{467}

468
469
470

\section{472
473}

\section{474
475}

\section{477}

\section{479}

479
480
481

\section{483}

\section{485}

\section{487
488}

\section{8}

490
49
492

\section{493}

\section{4}

\section{497
498}

\section{499
500}

\section{0 \\ 503
504}

(a)

507

\section{509}

510
51

\section{1}

\section{517
518}$$
\begin{aligned}
& 520 \\
& 52
\end{aligned}
$$

\section{522
523
524}

$$
\begin{aligned}
& 523 \\
& 524 \\
& 525 \\
& 526
\end{aligned}
$$

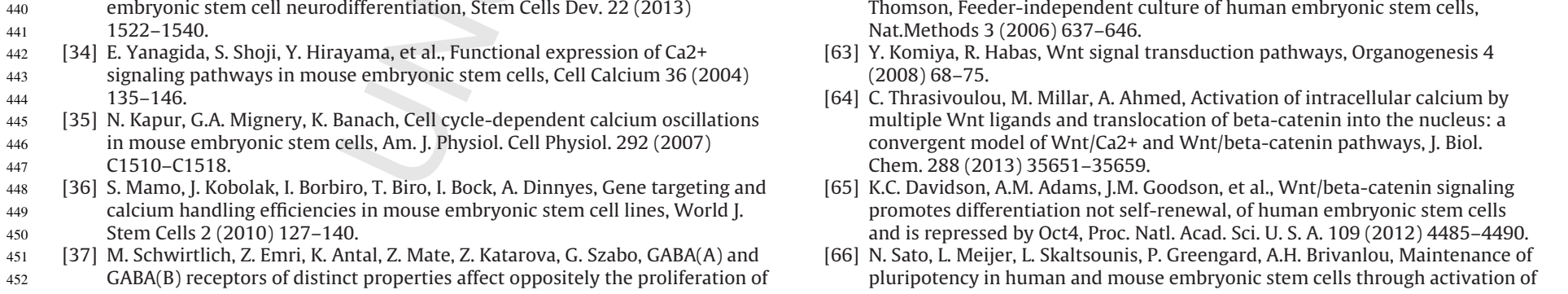

\footnotetext{
Please cite this article in press as: Á. Apáti, et al., Calcium signaling in human pluripotent stem cells, Cell Calcium (2016), http://dx.doi.org/10.1016/j.ceca.2016.01.005
} 
Wnt signaling by a pharmacological GSK-3-specific inhibitor, Nat Med 10 (2004) 55-63.

[67] T. Sumi, N. Tsuneyoshi, N. Nakatsuji, H. Suemori, Defining early lineage specification of human embryonic stem cells by the orchestrated balance of canonical Wnt/beta-catenin, Activin/Nodal and BMP signaling, Development 135 (2008) 2969-2979.

[68] A. Ermakov, S. Pells, P. Freile, et al., A role for intracellular calcium downstream of G-protein signaling in undifferentiated human embryonic stem cell culture, Stem Cell Res. 9 (2012) 171-184.

[69] C. Viero, O. Forostyak, E. Sykova, G. Dayanithi, Getting it right before transplantation: example of a stem cell model with regenerative potential for the CNS, Front. Cell Dev. Biol. 2 (2014) 36.

[70] G. Cocks, N. Romanyuk, T. Amemori, et al., Conditionally immortalized stem cell lines from human spinal cord retain regional identity and generate functional V2a interneurons and motorneurons, Stem Cell Res. Ther. 4 (2013) 69.

[71] F.M. Tonelli, A.K. Santos, D.A. Gomes, et al., Stem cells and calcium signaling, Adv. Exp. Med. Biol. 740 (2012) 891-916.

[72] J. Nichols, A. Smith, Naive and primed pluripotent states, Cell Stem Cell 4 (2009) 487-492.

[73] Q.L. Ying, J. Wray, J. Nichols, et al., The ground state of embryonic stem cell self-renewal, Nature 453 (2008) 519-523.
[74] T.W. Theunissen, B.E. Powell, H. Wang, et al., Systematic identification of culture conditions for induction and maintenance of naive human pluripotency, Cell Stem Cell 15 (2014) 471-487.

[75] O. Gafni, L. Weinberger, A.A. Mansour, et al., Derivation of novel human ground state naive pluripotent stem cells, Nature 504 (2013) 282-286.

[76] J. Hanna, A.W. Cheng, K. Saha, et al., Human embryonic stem cells with biological and epigenetic characteristics similar to those of mouse ESCs, Proc. Natl. Acad. Sci. U. S. A. 107 (2010) 9222-9227.

[77] J. Wang, G. Xie, M. Singh, et al., Primate-specific endogenous retrovirus-driven transcription defines naive-like stem cells, Nature 516 (2014) 405-409.

[78] C.B. Ware, A.M. Nelson, B. Mecham, et al., Derivation of naive human embryonic stem cells, Proc. Natl. Acad. Sci. U. S. A. 111 (2014) 4484-4489.

[79] G. Duggal, S. Warrier, S. Ghimire, et al., Alternative routes to induce naive pluripotency in human embryonic stem cells, Stem Cells 33 (2015) 2686-2698. 